\title{
Adiposis dolorosa as an etiology of abdominal pain
}

Sir,

Feurle recently discussed the classification, diagnosis, and treatment of abdominal wall pain [1]. Adiposis dolorosa is an important condition that should always be kept in mind when seeing a patient with abdominal pain of obscure origin. Adiposis dolorosa may produce severe, chronic pain that may lead to multiple diagnostic procedures and even surgery [2]. This condition usually appears in obese individuals, but rare cases have been reported in non-obese patients. It is characterized by localized pain within subcutaneous adipose tissue and rarely, pain within the abdomen simulating a more serious intra-abdominal process. Sometimes the pain is in a lipoma, but this is not necessary. The pain may be described as severe and prevents the patient from normal activities, including work. The absence of any other laboratory or radiographic abnormalities and lack of response to time and usual treatment often results in these patients being labeled as having anxiety disorder or psychological problems. The treatment of adiposis dolorosa is an intravenous infusion of 200-300 mg of lidocaine over a 30 minute period while the patient is attached to a cardiac monitor to detect possible lidocaine-induced cardiac arrhythmias. A high percentage of patients will respond temporarily or even permanently after one or more lidocaine infusions. Local infusions of lidocaine to the painful area are ineffective. The mechanism is unknown, but may be an effect on the central nervous system.

\section{References}

1. Feurle GE (2007) Abdominal wall pain - classification, diagnosis and treatment suggestions. Wien Klin Wochenschr 119 (21-22): 633-638

2. Atkinson RL (1982) Intravenous lidocaine for the treatment of intractable pain of adiposis dolorosa. Int J Obes 6 (4): 351-357

Correspondence: Richard L. Atkinson, MD, Obetech Obesity Research Center, 800 East Leigh St, \#50, Richmond, VA, USA, E-mail: ratkinson2@vcu.edu

\section{Author's reply}

It is correct that lipomatosis dolorosa, first described by Francis Xavier Dercum from Philadelphia in 1888, may be a differential diagnosis of abdominal wall pain. Careful palpation in Dercum's disease will, however, reveal not small pits as in abdominal wall disease but painful subcutaneous nodules. Further, the pain characteristic of these nodules does not increase when the muscles of the abdominal wall are contracted and these nodules also occur subcutaneously at the trunk and the extremities and rarely if ever exclusively under the skin of the abdominal wall. Clinical examination should therefore be able to distinguish these two entities.

Gerhard E. Feurle

Correspondence: Gerhard E. Feurle, MD, DRK Krankenhaus Neuwied, Marktstraße 104, 56564 Neuwied, Germany, E-mail: g.e.feurle@t-online.de 